\author{
MITSUBISHI ELECTRIC RESEARCH LABORATORIES \\ http://www.merl.com
}

\title{
QoS Harmonization for Home Networks
}

\author{
Ghulam Bhatti, Yige Wang, Jinyun Zhang
}

TR2010-061 April 2010

\begin{abstract}
A modern home could contain a large variety of devices and all of them should be able to work together so as to bring a rich experience to the users. Quality of service (QoS) is a critical factor for measuring user satisfaction. It represents the ability to provide different priorities based on the type of the data flows or to guarantee a certain quality of performance to a particular service. Different devices may follow different protocols and each protocol has its own QoS management scheme. These QoS schemes are not necessarily compatible with one another and the inconsistency will significantly impair their efficacy. In this paper, we propose a harmonized protocol for home networks, which unifies all other protocols and solves the inconsistency problem effectively.
\end{abstract}

IEEE Broadband Multimedia Symposium 2010

This work may not be copied or reproduced in whole or in part for any commercial purpose. Permission to copy in whole or in part without payment of fee is granted for nonprofit educational and research purposes provided that all such whole or partial copies include the following: a notice that such copying is by permission of Mitsubishi Electric Research Laboratories, Inc.; an acknowledgment of the authors and individual contributions to the work; and all applicable portions of the copyright notice. Copying, reproduction, or republishing for any other purpose shall require a license with payment of fee to Mitsubishi Electric Research Laboratories, Inc. All rights reserved.

201 Broadway, Cambridge, Massachusetts 02139 



\title{
QoS Harmonization for Home Networks
}

\author{
Ghulam Bhatti, Senior Member, IEEE, Yige Wang, Member, IEEE, Jinyun Zhang, Fellow, IEEE, \\ Fernando M. Matsubara, Kohtaro Asai, Shin Miura, and Shinji Akatsu
}

\begin{abstract}
A modern home could contain a large variety of devices and all of them should be able to work together so as to bring a rich experience to the users. Quality of service $(\mathrm{QoS})$ is a critical factor for measuring user satisfaction. It represents the ability to provide different priorities based on the type of the data flows or to guarantee a certain quality of performance to a particular service. Different devices may follow different protocols and each protocol has its own QoS management scheme. These QoS schemes are not necessarily compatible with one another and the inconsistency will significantly impair their efficacy. In this paper, we propose a harmonized protocol for home network, which unifies all other protocols and solves the inconsistency problem effectively.
\end{abstract}

Index Terms-DSCP, Home network, QoS

\section{INTRODUCTION}

$I^{n}$ $\mathrm{n}$ today's lifestyle, modern homes are furnished with a variety of media devices such as PCs, gateways, iPods, DVD recorders, HDTV sets, set-top boxes, wireless printers, etc. Many of these devices are traditionally isolated, lacking a strong inter-connectivity, and thus making it difficult to share data among different devices. Emerging home networking technologies aim at providing ways to connect and control home devices for the user to have a rich experience while offering tools and protocols for a convenient management of all the networked devices.

The architecture of a home network is shown in Fig. 1. It demonstrates two major roles that modern home networks are going to play in the years to come. First, a home network acts as an extension to the access network. From the access network, data streams, which belong to different protocols, enter the home through a home gateway and are then forwarded to their destinations over the home network. The second role of the home network is inter-connecting the consumer electronics devices in the home environment. The devices within the home network should be able to communicate to each other through the home gateway despite of what protocols they use. A home gateway may also act as a temporary repository for the contents downloaded, for example, at night to be watched later. The underlying infrastructure is expected to use Internet Protocol (IP) based technologies for content delivery.

Manuscript received September 23, 2009.

G. Bhatti, Y. Wang, J. Zhang are with Mitsubishi Electric Research Laboratory, Cambridge, MA 02139 USA (e-mail: gbhatti, yigewang, jzhang@merl.com).

F. M. Matsubara, K. Asai, S. Miura, and S. Akatsu are with Mitsubishi Electric Corporation, Tokyo 100-8310, Japan. (e-mail: Matsubara.Masami (a)eb.MitsubishiElectric.co.jp).
Some of the devices in the home network may work as an extension of the access network, for example devices configured remotely to support an acceptable level of transmission quality in home network primary domains described in [12]. The home networks can thus be operating in a bigger operational environment where the access networks have direct influence on the home networking environments. The two domains, therefore, may overlap, which may have profound implications for the design and performance of the home networks. There may be, for example, a contention for networking resources among the devices in the two domains. In order to guarantee the required bit rate, delay, jitter, packet dropping probability, etc. for specified services, users, or data flows, an appropriate quality of service (QoS) mechanism must be used for this situation.

Since the devices in the home network do not necessarily follow the same protocol, their QoS schemes are different as well. When several streams enter a home gateway at the same time, because of the inconsistency of QoS schemes, the home gateway has no way to decide on the relative priorities for data packets in these streams. As a result, the QoS mechanism of each device becomes useless. It is, therefore, important to harmonize all different protocols, currently competing for providing services to home users, for fair and optimal resource allocation in home networks.

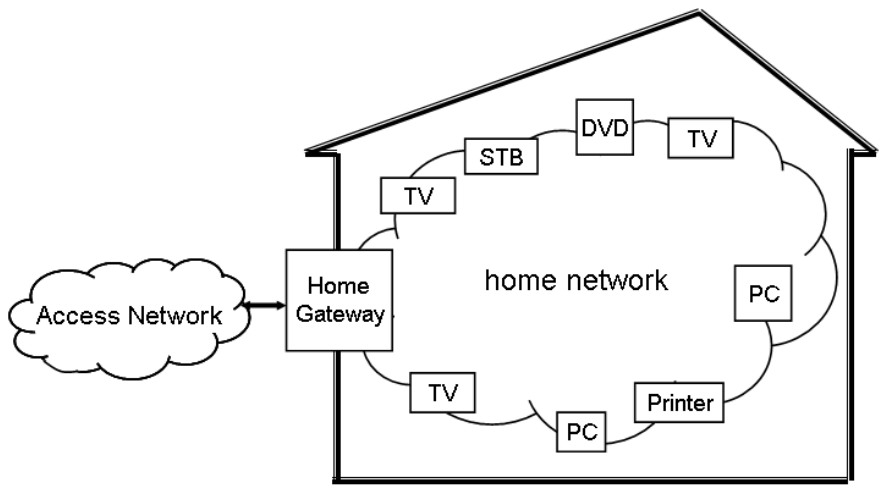

Fig. 1. Architecture of a home network.

In [2], the authors proposed to add an Intelligent Control Center (ICC) in the home network, which is placed between home gateway and all the other home devices. ICC controls all the upstream and downstream traffic and is regarded as an aggregation point. While the ICC, which has a central control, might be able to harmonize the inconsistencies in different QoS schemes, no specific mechanism for QoS harmonization was offered. In addition, because the harmonization process would take place in the ICC, the problem of optimal and fair resource allocation at the home gateway might still be left unaddressed 
for the streams that are originating from the access network. In [3], an IP Multimedia Subsystem (IMS) based scheme is proposed. This approach solves the mismatch problem between the access network and a home network if both use different QoS schemes. It can avoid the QoS conversion with the help of Session Description Protocol (SDP) in IMS networks. However, it has two problems. First, it depends on the IMS network and it is not known at this point if the real home networks will really make a use of the IMS. Secondly, since the QoS conversion does not harmonize among different QoS mechanisms, inconsistency still exists. In this paper, we propose adding two mini layers, called harmonization layers, to the IP stack within the home gateway. The harmonization layers, on one hand, are transparent to outside world and, on the other hand, they convert incompatible QoS schemes to a unified harmonized scheme so that the home gateway can determine priority levels without any confusion.

The rest of the paper is organized as follows. First we introduce QoS schemes for home network in Section II. Then we investigate several protocols and identify the QoS inconsistency among them in Section III. In Section IV, we propose a harmonization scheme for home network to solve the QoS inconsistency problem. Finally, we conclude the paper in Section V.

\section{QOS SCHEMES FOR HOME NETWORK}

As discussed in Recommendation ITU-T H.622 [12], currently two approaches are generally used for providing the required QoS: class-based QoS and session-based QoS.

In the class-based QoS approach, traffic is aggregated into a small number of classes, typically 4-8 classes, by tagging each data packet with a class ID. The packets having the same class ID, possibly belonging to different data streams, are given the same treatment at the network devices. In session-based QoS, a session is accepted (based on some admission control policy) and allowed to initiate only if sufficient resources to satisfy the requested QoS are available. Once a session is allowed, every terminal or application is required to reserve the necessary resources to support the desired QoS for the data stream. A specific signalling mechanism is used during the resource reservation mode. Once successful, the allocation/reservation of network resources is generally effective for the complete duration of that session.

While the class-based QoS scheme offers low complexity and high scalability, it does not guarantee the desired QoS. For the session-based scheme, if the resource reservation process has succeeded, the transmission quality of associated service is guaranteed for the duration of the session. However, session-based QoS has the following problems:

1. Some network devices are unaware of signaling protocol.

2. Network devices need to implement a complicated mechanism.

3. It results in higher latency because resource allocation process introduces additional session setup time.

A number of different technologies and standard Internet service protocols are known to provide class-based QoS in home networks. A short list of these protocols includes DSL-F TR-133 [7], DLNA [8], DVB-IP [9], and HGI [10]. Since harmonization among them is an item for further study in real world applications such as IPTV [16], in this paper we focus on a class-based QoS scheme.

For the services based on IP, a class-based QoS scheme differentiates priorities in the IP layer and data-link layer by using differentiated services code point (DSCP) in the IP header and QoS marking in the media access (MAC) header, respectively.

Differentiated Services (DiffServ or DS) is one of the mechanisms that are designed to support QoS requirements. The DiffServ specification has been defined by the Internet Engineering Task Force (IETF) in its Request for Comment (RFC) 2474 [13]. The key to DiffServ operation is a field within the IP header called the Differentiated Services (or DS) field, which consists of eight bits. The six most significant bits are called the DSCP while the remaining two bits are reserved for future use. With six bits available, a total of 64 DSCP values are available, thus allowing 64 standard Internet service protocol distinctions, or priority levels.

DiffServ-enabled routers and other network elements use the DSCP to differentiate the network traffic, i.e., classifying packets as these packets enter the local network. DiffServ uses the Behavior Aggregate (BA) classification method. In this classification method, packets are classified based only on the DSCP values. Packets from different sources having the same DSCP value are grouped as a BA and treated in the same manner.

For the data link layer, IEEE 802.11 [14] has four classes of priority, represented by AC_VO, AC_VI, AC_BE and AC_BK. Ethernet is a typical home network transmission medium. Prioritization of packets is provided as a part of Ethernet standard such as IEEE 802.1Q [4], IEEE 802.1D [6]. DSCP values can be mapped to corresponding data link layer QoS marking. Several protocols such as DLNA and HGI have provided mapping between DSCP and associated priority for data link layer QoS marking.

\section{PRoblem OF QoS SCHEMES}

It can easily be noticed that although all the protocols mentioned above adopt class-based QoS methods, a number of inconsistencies exist among them. For example, different protocols have a different number of priority levels, and the same DSCP value can represent different priority level in different protocols.

Table I summarizes some of these differences. The priorities for three commonly used protocols, DLNA, DVB-IP, and HGI are listed. The DSCP values with the same priority are in the same column. Note that the relative priorities given to different priority levels in these protocols just represent an example case. A different scaling can be used in actual deployments.

TABLE I

DSCP COMPARISON OF THREE PROTOCOLS

\begin{tabular}{|c|c|c|c|c|c|c|c|c|}
\hline & $\begin{array}{c}\text { Lower than } \\
\text { BE }\end{array}$ & $\mathrm{BE}$ & \multicolumn{6}{|c|}{ Higher than BE } \\
\hline DLNA & 0x08 & $0 \times 00$ & & & & $0 \times 28$ & & $0 \times 38$ \\
\hline DVB-IP & & $0 \times 00$ & $0 \times 1 \mathrm{~A}$ & $0 \times 24$ & $0 \times 22$ & $0 \times 2 \mathrm{E}$ & & \\
\hline HGI & $0 x 08$ & $0 \mathrm{x} 00$ & $0 \times 18$ & $0 \times 20$ & & $0 \times 28$ & $0 \times 30$ & $0 \times 38$ \\
\hline
\end{tabular}


From Table I, we can see that the protocols DLNA, DVB-IP, and HGI have 4, 5, and 8 priority levels, respectively, which do not match with one another. Moreover, the second highest priority (0x28) in DLNA, for example, is at the same overall level as the highest priority (0x2E) in DVB-IP and the third highest priority $(0 \times 28)$ in HGI.

It is worth mentioning that, due to various reasons, it is practically impossible to modify the protocols specified by various standards to get a consensus for a uniform priority scheme acceptable to all service protocols. On the other hand, a need for consistency and fairness necessitates a way where different data streams get correct resource allocation based on their relative priority level. In other words, the priorities need to be harmonized and determined consistently when streams using different protocols arrive at the home gateway (HG) without modifying existing protocols. That can only be achieved if a harmonization process takes place within the gateway device.

\section{HARMONIZATION LAYER-BASED QOS}

To harmonize QoS, we introduce two harmonization layers for a protocol stack implemented in the HG.

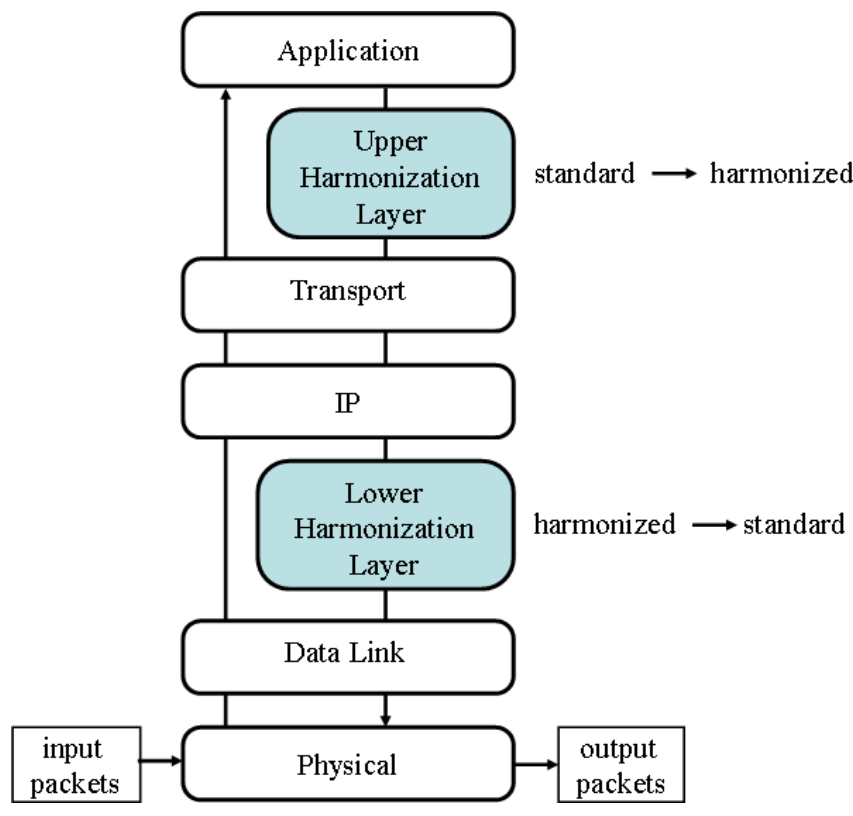

Fig. 2. Protocol stack including harmonization layers

Fig. 2 shows the layers of a protocol stack, which includes physical, data link, IP, transport, and application layers. The stack also includes an upper harmonization layer, and a lower harmonization layer. The upper harmonization layer resides between the application layer and a transport layer. The lower harmonization layer is placed between the IP layer and data link layer. The upper harmonization layer, by virtue of being interfaced with the application layer, can determine the priority level and the service protocol ID of each packet that passes through it. That information is crucial for the harmonization process.

Unlike conventional layers, the harmonization layers do not append (or delete) a header to (or from) the packets. Instead, they modify the IP header to implement the harmonization, which converts between standard priority levels and harmonized priority levels using a mapping table. This process of mapping and remapping is transparent to the access network and other devices in the home network.

Because different protocols have different QoS policies, the harmonization facilitates correct resource assignments to every stream according to its harmonized priority level. The harmonized priority level is applicable for all standard priority levels of all conventional Internet protocols.

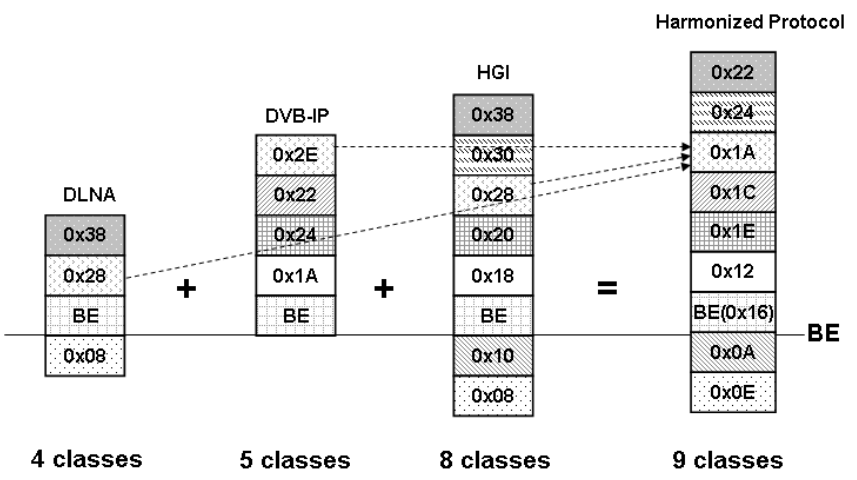

Fig. 3. Process of harmonizing protocols

Fig. 3 shows how to harmonize different protocols for three example conventional standard protocols DLNA, DVB-IP, and HGI. As shown in Fig. 3, for each protocol, the top class has the highest priority and the bottom class has the lowest priority. DLNA has three classes above best effort (BE) and one below BE. DVB-IP has four classes above BE, while HGI has five classes above $\mathrm{BE}$ and two below BE. Users or network administrators can select the priority relativity of different protocols. In Fig. 3, we follow the example priority scaling of Table I to determine the relative priority. In Fig. 3, the same hatching represents the same priority. Some classes of these three protocols have the same priority. For example, $0 \times 28$ in DLNA, 0x2E in DVB-IP, and 0x28 in HGI have the same priority. After merging different classes, a nine-class harmonized protocol is obtained. The classes of different protocols that have the same priority are converted to the same class in the harmonized protocol. For example, 0x2E in DVB-IP, $0 \times 28$ in HGI and DLNA are converted to $0 \times 1 \mathrm{~A}$ in the harmonized protocol. The definition of DSCP values and their relative priority levels in the harmonized protocol complies with the Assured Forwarding (AF) per-hop behavior (PHB) in RFC 2597 [15]. Because two classes in the harmonized protocol are below BE, an appropriate DSCP value for the BE class should be selected so that the DiffServ can determine the relative priority. In Fig. 3, we select 0x16 for BE.

For harmonization, some a priori information is stored in the memory of the HG as shown in Fig. 4. This information includes a harmonization mapping table that converts the DSCP value of any protocol to that of the harmonized protocol. Because some devices in home network cannot interpret the DSCP values in the IP header, the devices need to use the layer-2 QoS marking in the MAC header. Therefore, for each protocol, a DLL QoS mapping table, which converts the DSCP value to QoS marking of DLL, is also included. An ID mapping 
table is used to find the corresponding protocol identification of a packet. The memory also stores a QoS policy.

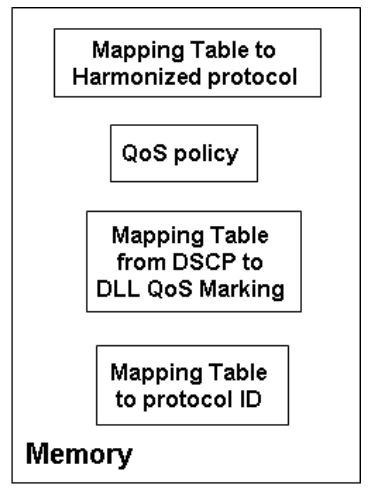

Fig. 4. Memory in the home gateway

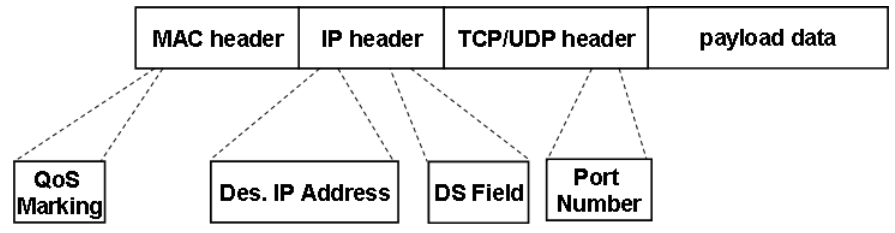

Fig. 5. Structure of an Internet packet.

The protocol ID can be decided based on information in headers of the Internet packet. Fig. 5 shows a conventional Internet packet. The packet includes a media access control (MAC) header, an IP header, TCP/UDP header, and payload data. Fields of interest are the QoS marking in the MAC header, the destination IP address and the DS field in the IP header, and the port number in the TCP/UDP header. Table II shows an example of the ID mapping table. In Table II, there are three columns, which are destination IP address, destination port number and protocol. For any stream, when the destination IP address and port number are known, the corresponding protocol is determined from this Table.

TABLE II

MAPPING TABLE TO PROTOCOL ID

\begin{tabular}{c|c|c}
\hline \hline Des. IP Address & Des. Port No. & Protocol ID \\
\hline 201.133 .25 .10 & 99 & DLNA \\
201.133 .25 .12 & 10 & DVB-IP \\
201.133 .25 .16 & 25 & DLNA \\
201.133 .25 .11 & 5 & HGI \\
201.133 .25 .18 & 20 & DVB-IP \\
\hline \hline
\end{tabular}

An example of mapping table from DSCP values to data link layer QoS marking for HGI is shown in Table III.

Table IV shows an example of a mapping table that converts the DSCP value of any protocol to that of the harmonized protocol. The columns in the table are the protocol the stream belongs to, the original DSCP value, and the harmonized DSCP value based on the harmonized protocol, respectively. In practice, the mapping table has entries for all different protocols that need to be harmonized.
TABLE III

MAPPING TABLE FROM DSCP TO DLL QOS MARKING

\begin{tabular}{c|c|c}
\hline \hline DSCP & 802.11 & $802.1 \mathrm{D}$ \\
\hline 0x38 & AC_VO & 7 \\
0x30 & AC_VO & 6 \\
0x28 & AC_VI & 5 \\
0x20 & AC_VI & 4 \\
0x18 & AC_BE & 3 \\
BE (0x00) & AC_BE & 0 \\
0x10 & AC_BK & 2 \\
0x08 & AC_BK & 1 \\
\hline \hline
\end{tabular}

TABLE IV

MAPPING TABLE TO HARMONIZED PROTOCOL

\begin{tabular}{c|c|c}
\hline \hline Protocol & DSCP & $\begin{array}{c}\text { Harmonized DSCP of } \\
\text { Harmonized Protocol }\end{array}$ \\
\hline DLNA & $0 \times 38$ & $0 \times 38$ \\
DLNA & $0 \times 28$ & $0 \times 28$ \\
DLNA & BE $(0 \times 00)$ & BE \\
DLNA & $0 \times 08$ & $0 \times 08$ \\
DVB-IP & $0 \times 2 E$ & $0 \times 28$ \\
DVB-IP & $0 \times 22$ & $0 \times 22$ \\
DVB-IP & $0 \times 24$ & $0 \times 20$ \\
DVB-IP & $0 \times 1 \mathrm{~A}$ & $0 \times 18$ \\
DVB-IP & BE $(0 \times 00)$ & $\mathrm{BE}$ \\
HGI & $0 \times 38$ & $0 \times 38$ \\
HGI & $0 \times 30$ & $0 \times 30$ \\
HGI & $0 \times 28$ & $0 \times 28$ \\
HGI & $0 \times 20$ & $0 \times 20$ \\
HGI & $0 \times 18$ & $0 \times 18$ \\
HGI & BE $(0 \times 00)$ & BE \\
\hline \hline
\end{tabular}

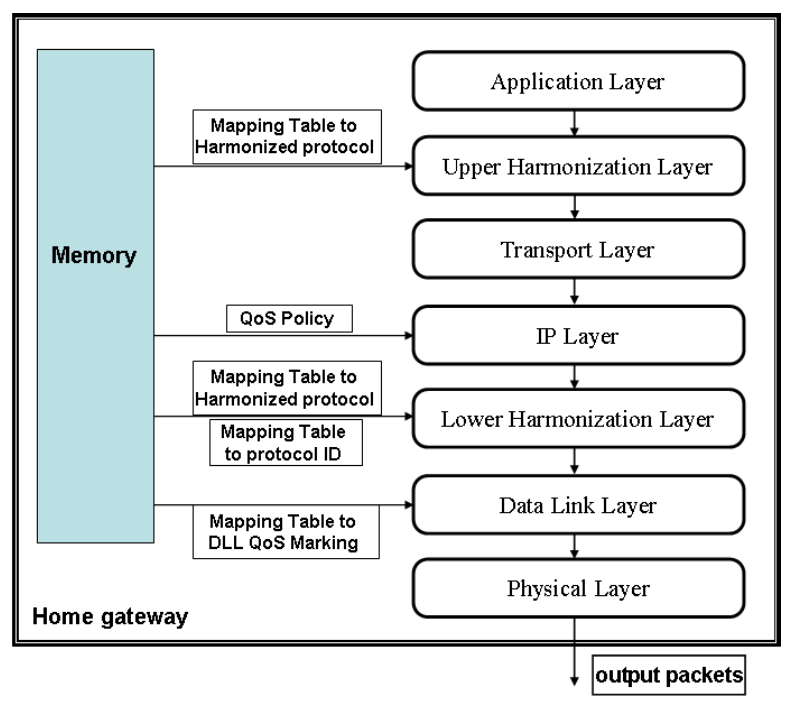

Fig. 6. Process of harmonizing protocols

Fig. 6 shows the harmonization process in the HG. The harmonization process is only applied to down stream traffic, i.e., the packets passed from the application layer to the physical layer. For a packet, the application layer generates the DSCP value, based on, for example, the type of subscription, service, and protocol. The application layer passes the DSCP value and service protocol ID along with other pertinent information, such as destination IP address and port number, 
etc., to the upper harmonization layer. The upper harmonization layer uses the harmonization mapping table to generate a harmonized DSCP value. The original DSCP value is replaced by the harmonized DSCP value. The IP layer follows the DiffServ QoS policy to fulfill QoS requirements according to the harmonized DSCP value.

The stream passes to the lower harmonization layer, which uses ID mapping table to obtain the protocol ID for the stream. Then, based on harmonization mapping table, an inverse conversion is performed and the original DSCP value of the stream is recovered. The harmonized DSCP value is replaced with the original value. When the stream arrives at the data link layer, according to the DLL QoS mapping table, the QoS marking of the DLL is obtained and written into the MAC header.

In the IP layer, based on the harmonized DSCP values, streams enter different buffering queues. Every queue is allocated a different set of resources according to its priority level. Streams with the same priority level but using different protocols will be placed into the same queue despite of their different DSCP values originally assigned to them. Then every outgoing data frame gets an appropriate treatment according to its harmonization priority level.

Fig. 7 shows the queuing process of the IP layer. In our example, the harmonized protocol has nine priority levels or classes. Therefore, there are nine corresponding queues. Queue 1 has the highest priority and queue 9 has the lowest priority. When the input packet enters the IP layer, the packet is queued according to the QoS policy based on the harmonized DSCP value.

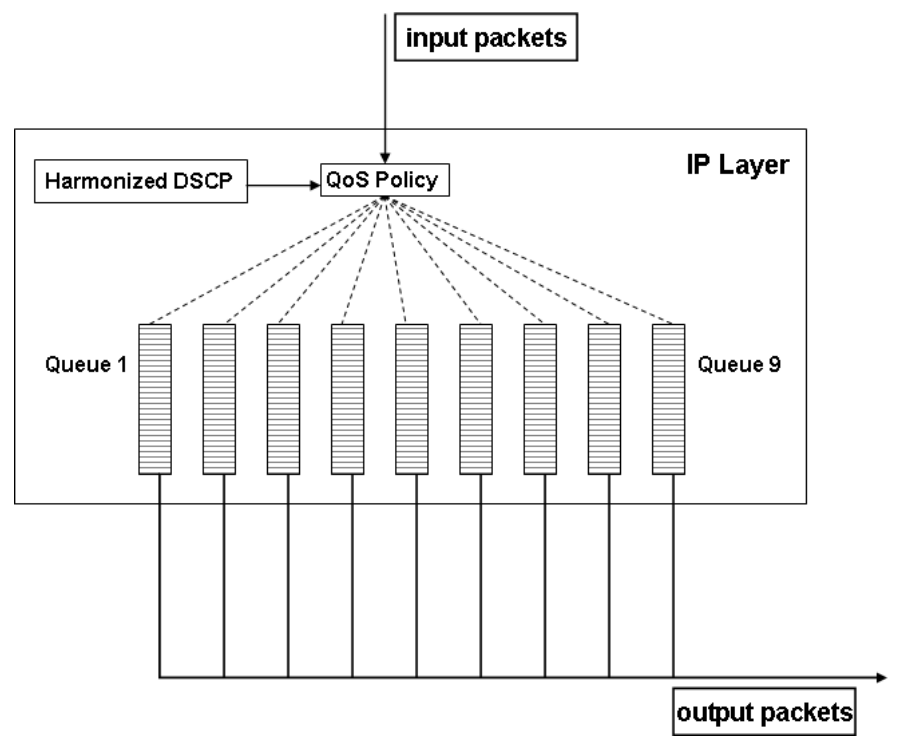

Fig. 7. Process of queuing process of IP layer

\section{CONCLUSION}

In this paper, we proposed an approach to harmonize the QoS schemes for different service protocols used for streaming data into home networks. By preserving the relative priorities used by different service protocols, the proposed method can effectively solve the QoS inconsistency problem in home networks, facilitate optimal resource allocation, and improve overall user experience.

\section{REFERENCES}

[1] ITU-T Series H, Supplement 11, "Analysis of class-based home network QoS solutions".

[2] G. Liu, S. Zhou, X. Zhou, and X. Huang, "QoS Management in Home Network," in Int. Conf. Computational Intelligence for Modelling, Control and Automation, and Int. Conf. Intelligent Agents, Web Technologies and Internet Commerce, Nov. 28, 2006.

[3] S. L. Tompros, C. D. Kavadias, D. D. Vergados, and N. P. Mouratidis, "A Strategy for Harmonised QoS Manipulation in Heterogeneous IMS Networks," Wireless Personal Communications, vol. 49, no. 2, pp. 197-212, Apr. 2009.

[4] IEEE 802.1Q, IEEE standard for local and metropolitan area networks virtual bridged local area networks.

[5] IETF RFC 2474, Definition of the Differentiated Services Field (DS Field) in the IPv4 and IPv6 Headers.

[6] IEEE 802.1D (2004), IEEE Standard for Local and metropolitan area networks Media Access Control (MAC) Bridges.

[7] DSL Forum TR-133 (2005), DSLHome TR-064 Extensions for Service Differentiation.

[8] IEC 62481-1 (2007), Digital living network alliance (DLNA) home networked device interoperability guidelines - Part 1: Architecture and protocols.

[9] ETSI TS 102034 (2007), Digital Video Broadcasting (DVB); Transport of MPEG-2 TS Based DVB Services over IP Based Networks.

[10] Home Gateway Initiative (2006), Home Gateway Technical Requirements: Release 1.0.

[11] UPnP QoS (2006), UPnP QoS Architecture: 2.

[12] Recommendation ITU-T H.622 (2008), A generic home network architecture with support for multimedia services.

[13] RFC 2474, Definition of the Differentiated Services Field (DS Field) in the IPv4 and IPv6 Headers.

[14] IEEE 802.11 (2007), IEEE Standard for information technology -Telecommunications and information exchange between systems Local and metropolitan area networks - Specific requirements Part 11: Wireless LAN Medium Access Control (MAC) and Physical Layer (PHY) Specifications.

[15] RFC 2597, Assured Forwarding PHB Group.

[16] Recommendation ITU-T H.622.1 (2008), Architecture and functional requirements for home networks supporting IPTV services. 